\title{
Impact of prophylactic bilateral salpingectomy on ovarian reserve in women undergoing vaginal hysterectomy: A randomized controlled trial
}

Article

\author{
Ashraf Nassif Mahmoud Elmantwe ${ }^{1}$, Mohamed Anwar Elnory ${ }^{2}$ \\ Department of Obstetrics and Gynecology, Faculty of Medicine, Benha University, Egypt
}

\begin{abstract}
Aim: The purpose of this study was to evaluate whether bilateral opportunistic salpingectomy (BOS) in premenopausal women has any detrimental effect on ovarian reserve $(\mathrm{OR})$ and if this increases surgical consequences in women undergoing vaginal hysterectomy (VH)/Non-Descent vaginal hysterectomy (NDVH).

Materials and Methods: This prospective, open-label, randomized, multisite, parallel group, concealed allocation, superiority trial was conducted at Benha University Hospital (BUH), and two private centers in El-Qalubia, Egypt, From September 2015 to September 2017. 110 women undergoing VH/NDVH were allocated to adding BOS (intervention group) or not (control group) at $1: 1$ ratio. The primary outcomes were differences in change of serum Anti-müllerian hormone $(\triangle \mathrm{AMH})$, serum follicular stimulating hormone $(\triangle \mathrm{FSH})$, antral follicular count $(\triangle \mathrm{AFC})$, flow index $(\Delta \mathrm{FI})$ vascularization index $(\Delta$ VI), vascularization flow index $(\Delta$ VFI) and calculated ovarian age with OvAge $(\Delta$ OvAge), measured preoperatively and at 6 to 8 months postoperatively. The secondary outcomes were surgical outcomes as well as the feasibility of performing BOS at VH/NDVH and hospital stay.

Results: Baseline demographic, clinical, hormonal and three-dimensional ultrasound characteristics did not show significant differences between both groups according to both intention to treat (ITT) analysis as well as per protocol (PP) analysis. Also, the groups did not differ significantly regarding operative outcomes such as operative time, operative bleeding and hospital stay according to ITT and PP analyses. The BOS was surgically feasible in 95\% (58/61) of tried cases. In both groups the postoperative AMH, AFC, VI, FI, VFI were decreased, while FSH, OvAge were increased. There were no statistically significant differences between intervention and control groups according to ITT as well as PP analyses regarding $\triangle \mathrm{AMH}(P=0.17), \Delta \mathrm{FSH}(P=0.11), \triangle \mathrm{AFC}(P=0.07), \Delta \mathrm{VI}(P=0.82), \Delta \mathrm{FI}(P=0.94)$,

$\Delta$ VFI $9(P=0.96)$ and $\triangle$ OaAge $(P=0.78)$.

Conclusion: Performing bilateral opportunistic salpingectomy at time of vaginal hysterectomy / Non-descent vaginal hysterectomy did not have a detrimental effect on ovarian reserve as well as surgical consequences when compared with a policy of performing hysterectomy alone. So adding BOS as ovarian cancer risk-reducing surgery appears to be a safe procedure in average OvCa risk premenopausal women undergoing VH/NDVH for benign gynecological indications.
\end{abstract}

Key Words: Anti-müllerian hormone, bilateral opportunistic salpingectomy, non-descent vaginal hysterectomy, ovarian cancer, ovarian reserve, vaginal hysterectomy

Received: 3 January 2020, Accepted: 24 January 2020

Corresponding Author: Ashraf Nassif Mahmoud Elmantwe, Department of Obstetrics and Gynecology, Faculty of Medicine, Benha University, Egypt,Tel.: 01001801244, E-mail: shrfnassif@gmail.com

ISSN: 2090-7265, May 2020, Vol.10, No. 2

\section{INTRODUCTION}

The lifetime risk for developing ovarian cancer $(\mathrm{OvCa})$ in developed world is $\approx 1.4 \%^{[1-3]}$, and this could be rounded to $\approx 1 \%$ for developing countries $^{[2]}$, of them up to $45 \%$ only whom their 5 year survival is $\geq 5$ years $^{[1,3]}$, this, despite great advance in OvCa treatment. Researchers continue in introducing evidences regards epithelial ovarian cancers (EOCs) especially high grade serous ovarian carcinomas (HGSOCs), as well as primary peritoneal carcinoma (PPC), that is EOCs originated in fallopian tube (FT), especially the lateral fimbrial ends as a serous tubal intraepithelial carcinoma
(STIC), which later on incorporated into ovarian surface epithelium ${ }^{[4-8]}$. Due to this recently introduced theory regards ovarian tumorigenesis and owing to lack of effective OvCa screening programs, a paradigm shift in $\mathrm{OvCa}$ management directed at primary surgical prevention through the prophylactic removal of FT in women completing their reproductive desire in average OvCa risk women ${ }^{[4]}$. This concept was first introduced in September 2011 by the society of gynecologic oncology (SGO) of Canada ${ }^{[10]}$ and followed by SGO of United States (USA) in November 2013 ${ }^{[11]}$ then American colleges of Obstetrics and Gynecology (ACOG) in 2015 ${ }^{[12]}$ then taken globally by gynecologist ${ }^{[13-17]}$. 
Meta-analysis (MA) and systematic reviews $(\mathrm{SRs})^{[16,17]}$ reviewed Sweden's cohort ${ }^{[18]}$ as well as the $\mathrm{USA}^{[19]}$ and Denmark ${ }^{[20]}$ case-controlled populationbased studies including 3509 women who undergoing indicated bilateral salpingectomy (BS) and 5.655.702 controls who did not experience BS and they found ${ }^{[16]}$ $\mathrm{BS}$ significantly reduces the risk of $\mathrm{OvCa}$ occurrence in women underwent $\mathrm{BS}$ relative to controls $(\mathrm{OR}=0.51,95 \%$ CI $0.35 \% 0.75, \mathrm{I}^{2}=0 \%$ ). Despite the theoretical benefits of prophylactic bilateral salpingectomy (PBS) in reducing OvCa risk (so the name of risk-reducing salpingectomy $(\mathrm{RRS}))^{[9]}$ isn't reported in prospective trial till now ${ }^{[16,17]}$ and there is only ongoing one registered trial on clinical trial.gov (NCT 03045965) from Sweden. This trial evaluates impacts of adding bilateral opportunistic salpingectomy (BOS) in women undergoing hysterectomy through all routes on surgical complications, menopausal symptoms onset, ovarian functions and risk of $\mathrm{OvCa}$ over a 10 - to 30 year follow-up period (https://clinicaltrials.gov/CT2/show/NCT03045965) ${ }^{[21]}$.

Hysterectomy is the $2^{\text {nd }}$ commonly performed gynecological procedures in women after cesarean section worldwide ${ }^{[22]}$. Hysterectomy routes include abdominal (AH, TAH), laparoscopic (TLH, LAVH) and vaginal $(\mathrm{VH})^{[22]}$. Despite that $\mathrm{SRs}^{[22]}$ of randomized controlled trials (RCTs) have been demonstrated that $\mathrm{VH}$ is the preferred route for hysterectomy especially for Benign indications, the TAH, followed by TLH, LAVH remains the commonly utilized routes ${ }^{[22]}$. $\mathrm{VH}$ usually performed for women with pelvic organ prolapse (POP) with $\geq$ stage 2 on POPQ, despite that $\mathrm{VH}$ might be performed in all indications for hysterectomy with a meager rate of conversion to others routes ${ }^{[23]}$. The low utilization rates of non-descent vaginal hysterectomy (NDVH) ${ }^{[23-25]}$ might be increased, if BOS considered to be the standard policy at hysterectomy rather than a recommendation on which the route shouldn't be changed ${ }^{[12,26]}$. This low rate of VH/NDVH exist and might continued, despite recently published studies on high success rates of BS at $\mathrm{VH}^{[23,24,27]}$ as well as recently introduced elegant decision analysis model developed by Cadish et al..$^{[28]}$ on efficacy of BOS at $\mathrm{VH}$ based on published literature and USA national surgical quality improvement program database. Cadish et al. ${ }^{[28]}$ found adopting the policy of BOS at $\mathrm{VH}$ will reduce $\mathrm{OvCa}$ cases from $0.95 \%$ to $0.511 \%$ $(\mathrm{NNT}=1 / 225)$ and $\mathrm{OvCa}$ deaths from $0.478 \%$ to $0.256 \%$ $(\mathrm{NNT}=1: 450)$. Studies to date, evaluates impacts of adding BOS to hysterectomy includes abdominal route $(\mathrm{TAH}, \mathrm{AH})^{[29]}$ as well as laparoscopic routes $(\mathrm{TLH}, \mathrm{LAVH})^{[30-34]}$ or both ${ }^{[35]}$ but no trial up till now evaluates the impact of VH / NDVH \pm BOS (PBS) (RRS) on ovarian reserve $(\mathrm{OR})$ and ovarian functions $(\mathrm{OF})$.

\section{AIM OF THE WORK}

The objective of this trial was to evaluate the impact of $\mathrm{VH} / \mathrm{NDVH}$ alone versus VH/NDVH with BOS (PBS) on ovarian reserve parameters as well as the feasibility of PBS at time of VH/NDVH and its surgical consequences.

\section{PATIENTS AND METHODS}

We did this prospective, parallel group, randomized, concealed allocation, open-label, superiority trial at 3 surgical sites including Benha University Hospital Obstetric and Gynecology Department, El-Hayat Obstetric \& Gynecology Specialized center (both in Benha City) as well as Al Taqwa specialized Hospital in Abu Zabal, all centers are in Al-Qalubia Governorate, Egypt from September 2015 to September 2017. We gained ethical approval for this study protocol from Benha Faculty of Medicine ethical committee. Also, all enrolled participants signed informed written consents. All women scheduled for VH/NDVH due to benign indications and deemed feasible for the vaginal route of hysterectomy between September 2015 and September 2017 were asked to participate in this trial.

Eligible participants were American Society of Anesthesiologist (ASA) physical status I-II, premenopausal aged between 35 - 50 years old, with benign indications for hysterectomy including POP, dysfunctional uterine bleeding (DUB), fibroids, premalignant changes in the cervix, endometrium as cervical intraepithelial neoplasia (CIN), endometrial hyperplasia (EH). Prior pelvic surgery like a cesarean section was not considered exclusion from this trial. We excluded from this trial women with anticipated adnexal pathologies as an ovarian cyst and hydrosalpinx as well as women at high risk for developing $\mathrm{OvCa}$ as those with current or history of breast cancer (BC), family history of $\mathrm{BC}, \mathrm{OvCa}$, known BRCA I, II mutations families. Also, women with age range between 35 - 50 year who had symptoms suggestive of menopausal onset were excluded. Additional exclusion criteria include women who received estrogen-progestin therapy in prior three months, women with acute or chronic pelvic inflammatory disorders (PIDs), malignant gynecologic neoplasm, prior chemotherapy, radiotherapy, autoimmune diseases, chronic endocrinal diseases as hyperprolactinemia, congenital adrenal hyperplasia, hypogonadotropic hypogonadism, also women uncontrolled diabetes mellitus.

We recruited participants in this trial sequentially, and we allocated them to $\mathrm{VH} / \mathrm{NDVH}$ alone (control group) or $\mathrm{VH} / \mathrm{NDVH}$ with $\mathrm{PBS}$ (intervention group) randomly at a $1: 1$ ratio. The trial statistician created a randomized treatment allocation schedule of a different block size, where POP $\geq$ stage II on POPQ, uterine fibroids $\geq 14$ weeks, prior pelvic surgeries, body mass index BMI > $30 \mathrm{~kg} / \mathrm{m} 2$ were blocking items. The randomization list created by random computer generator and stored by the gynecologist (M.A.E). The point of randomization was on the day before planned surgical procedures. After randomization, the investigators, as well as participants, knew the assignment group but both radiologist, as well 
as the clinical pathologist, remains blinded to group assignment. The surgical procedures have taken place as preferred by the gynecologist (MAE and ANME), in the group assigned to the intervention group, FT either removed separately after uterine removal (Fig. 1a) or on continuity with the uterus (Fig. 1b,c).
We used different surgical technique including clamping, cutting, suturing of mesosalpinx as well as monopolar and bipolar electrocautery (Fig. $1 \mathrm{~d}$ ) to do such task after securing round and utero-ovarian ligaments. We take care not to injure the ovarian blood supply from the infundibulopelvic (IP) ligament.

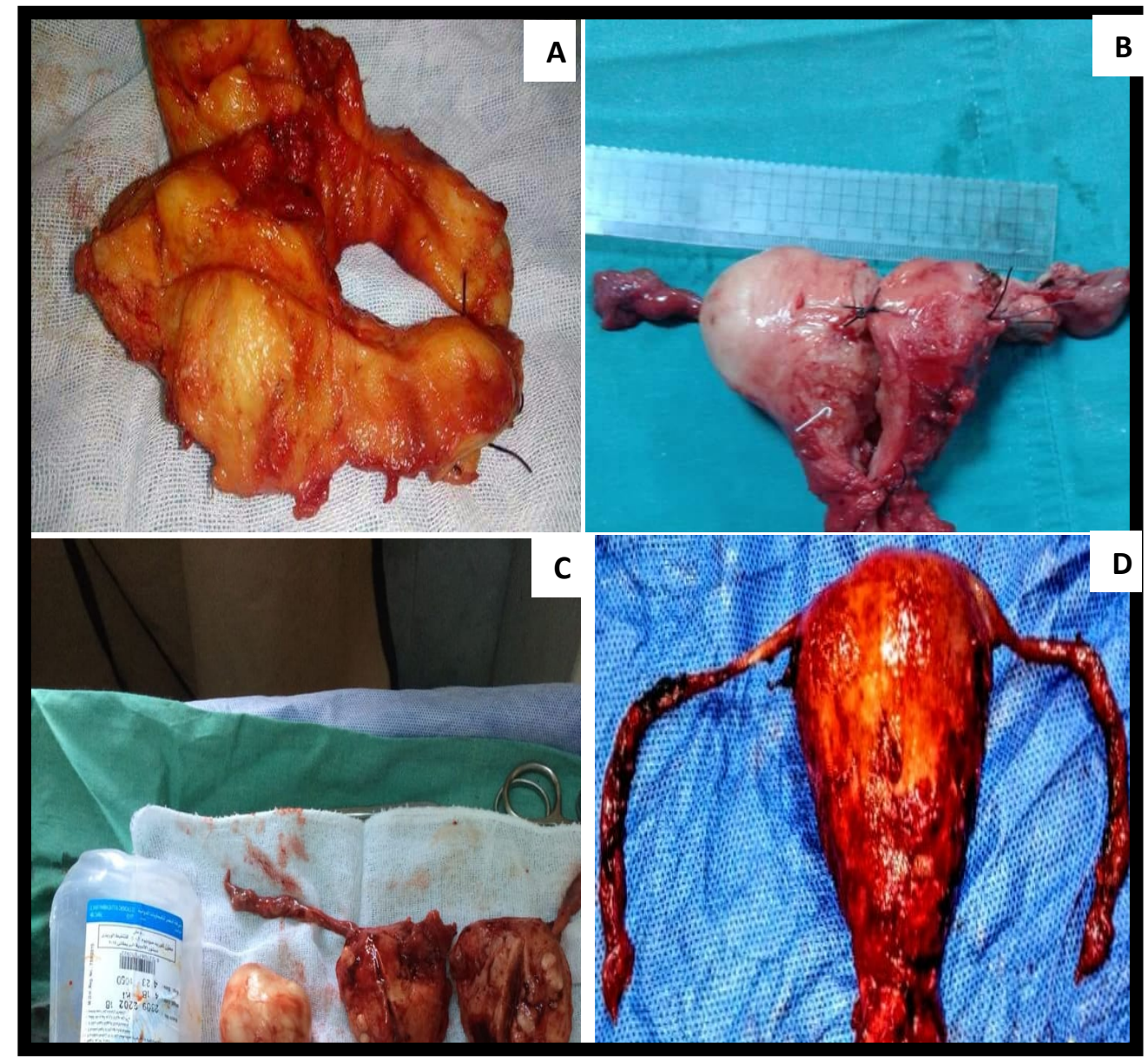

Fig.1: Vaginal Hysterectomy (VH)/Non-Descent Vaginal Hysterectomy (NDVH) with bilateral opportunistic salpingectomy(BOS) specimens A) NDVH without BOS, B) NDVH with BOS removed after uterine removal, C) Bisected NDVH with myomectomy and BOS in continuity, D) Pelvic organ prolapse of $\geq$ stage 3 POPQ with BOS in continuity removed with monopolar cautery.

Participants included in this trial were assessed in between day 1 and day 4 in the cycle where $\mathrm{VH} /$ NDVH was performed as well as at expected early follicular phase between 6 and 8 months postoperatively. We assessed this trial cohort regards changes $(\Delta)$ in anti-müllerian hormone (AMH), follicular stimulating hormone (FSH), three-dimensional (3DUS) antral follicular count (AFC), flow index (FI), vascular index (VI) and vascular flow index (VFI). On discharge and after six months from hysterectomy all trial participants were asked to return back, after 6 to 8 months at time, they felt release from premenstrual tension. The returned back participants were evaluated by conventional transvaginal ultrasound (TVS) and if women found to have dominant follicle $(>10 \mathrm{~mm})$ or ovarian cyst, we asked them to return back after 15 - 20 days. We sent participant to three-dimensional TVS examination and blood sampling for hormonal analyses, if we found their ovaries in quiescent state.

Blood samples were taken via venipuncture and were centrifuged at $3500 \mathrm{rpm}$ for 10 minutes within 30 minutes maximally to 4 hours stored at $4^{\circ} \mathrm{C}$. Serum samples Aliquots were frozen at $-80^{\circ} \mathrm{C}$ to the time of when we collected all samples. AMH was measured by an AMH Gen II ELISA assay kit (Beckman Coulter / USA) with sensitivity limits of $0.16 \mathrm{ng} / \mathrm{ml}$ and inter-intra assays variation coefficients of $\leq 0.4 \%$ and $3.4 \%$, respectively. FSH was measured by an ECLIA method on COBASe 411 autoanalyzer (Roche Diagnostics, Germany) with detection limits of $0.100 \mathrm{Iu} / \mathrm{ml}$ and inter-intra assays variation coefficients of $\leq 3.5 \%, 2.6 \%$, respectively. 
On the same day of blood sampling women were examined by single sonographer on volusone pro 370 (GE healthcare ultrasound, Switzerland) with transvaginal 3D ultrasound scanning modes assessing both AFC as well as 3D vascularization indices (VI, FI, VFI) with aids of sonography based automated volume count and virtual organ computer-aided analysis imaging programs (SonoAVCTM and VOCAl TM, GE, healthcare ultrasound, Switzerland). The mean $(95 \% \mathrm{CI})$ of intra-class correlation coefficient (ICG) (as a measure for intra-observer reliability) were 0.97 (0.86 - 0.98), 0.97 (0.96 - 0.99), $0.88(0.71-0.97)$ and 0.99 (0.97 - 0.99), respectively, for ovarian volume (OV), VI, FI and VFI. The mean $(95 \%$ CI) of ICCs for data acquisition for VFI, FI, VI, and OV were 0.98 (0.95 - 0.99), 0.98 (0.96 - 0.99), 0.98 (0.94 - 0.99) and $0.99(0.96-0.99)$, respectively.

The main outcomes of this trial were evaluating impacts of adding BOS (PBS) (RRS) to VH / NDVH on $\mathrm{OR}$ and $\mathrm{OF}$. We accessed for this aim the simple changes ( $\Delta=$ postoperative level at 6 to 8 months preoperative level) in $\mathrm{AMH}, \mathrm{FSH}, \mathrm{AFC}, 3 \mathrm{D}$ vascularization indices (VI, FI, VFI) as well as considering the change in OvAge test suggested by Venturella et al. ${ }^{[35,36]}$. Where OvAge $=$ years corresponds to chronological age in healthy female and calculated from generalized linear formula $=48.05-(3.14) \times \mathrm{AMH}+(0.07) \times \mathrm{FSH}-0.77$ $\mathrm{x} \mathrm{AFC}-(0.11) \mathrm{x} \mathrm{FI}+(0.25) \mathrm{x} \mathrm{VI}+(0.1) \mathrm{x} \mathrm{AMH} \mathrm{x}$ $\mathrm{AFC}+0.02 \times \mathrm{FSH} \times \mathrm{AFC}{ }^{[35,36]}$. The subsidiary outcomes of this trial were achievability of adding $\mathrm{BS}$ to $\mathrm{VH} /$ NDVH as well as operative times, blood loss, change in hemoglobin $(\triangle \mathrm{HB}=\mathrm{HB}$ preoperatively-HB at discharge from surgical center), hospital stay, surgical consequences were defined as any event the required (re) intervention or prolonged hospital stay.

A sample size per treatment arm of 44 women were considered adequate to detect a clinically significant absolute difference in $\triangle$ AMH $(6$ to 8 months postoperative - preoperative) of $0.3 \mathrm{mcg} / \mathrm{L}(\mathrm{ng} / \mathrm{ml})^{[37]}$ between interventional and control groups using unpaired student t-test at $80 \%$ power and double side significance of $0.05 \%$. As, we expect a dropped rate approximately of $25 \%$ due to the long period between initiation and termination of the trial, a total of 55 women needed per group.

\section{STATISTICAL ANALYSIS:}

We used SPSS package for Windows (SPSS, version 24, IBM SPSS statistics, IBM Corp. released 2016, Chicago, IL, USA) for statistical analysis. We performed all analysis according to intention-totreat (ITT) as well as per-protocol (PP) analysis. As our continuous variables were normally distributed, we presented them as means, standard deviations and ranges as well as we tested for significance between them by unpaired student t-test. We presented categorical data as numbers and percentages while we used Fisher's exact test to determine the significance between them. We assessed significance by point estimate difference with $95 \%$ confidence interval as well as setting $P$ value at $<0.05$.

\section{RESULTS}

As presented in figure 2, we evaluated 150 women for suitability to be chosen in this trial. One hundred and ten women were suitable, consented to participate in this study and randomized to either $\mathrm{VH} / \mathrm{NDVH}$ without BOS (control group) or $\mathrm{VH} / \mathrm{NDVH}$ with BOS (intervention group), fifty-five participants in each group. After randomization and before surgery one patient in the intervention group and two patients in control group were dropped as well as two women were canceled before surgery, one in each group due to hypertension and high titer PCR test for hepatitis $\mathrm{C}$ virus.

In intervention group, three women after consented for BOS required just before the surgery to conserve their tubes despite careful counseling (they were feared from impact of BOS on ovarian functions), as well as one women converted to TAH with BOS due to difficulty in extraction of uterine specimen and in 3 women we couldn't perform BOS after completing NDVH owing to extensive adhesion while we could perform VH/NDVH with BOS as allocated in 46 women.

In control group, 14 women after consented to be included in control arm of this trial, requested to perform BOS for fear of ovarian cancer, despite careful counseling regarding that this benefit is still a theoretical benefit, as well as 2 cases converted to TAH without BOS due to lack of mobility of sizable uteri and 2 cases in whom TLH without BSO were performed as requested by senior staff while 34 women received $\mathrm{VH} / \mathrm{NDVH}$ as allocated. We completely followed up 48 women and 46 women. While, we lost to follow up five women and six women in intervention and control group, respectively. 


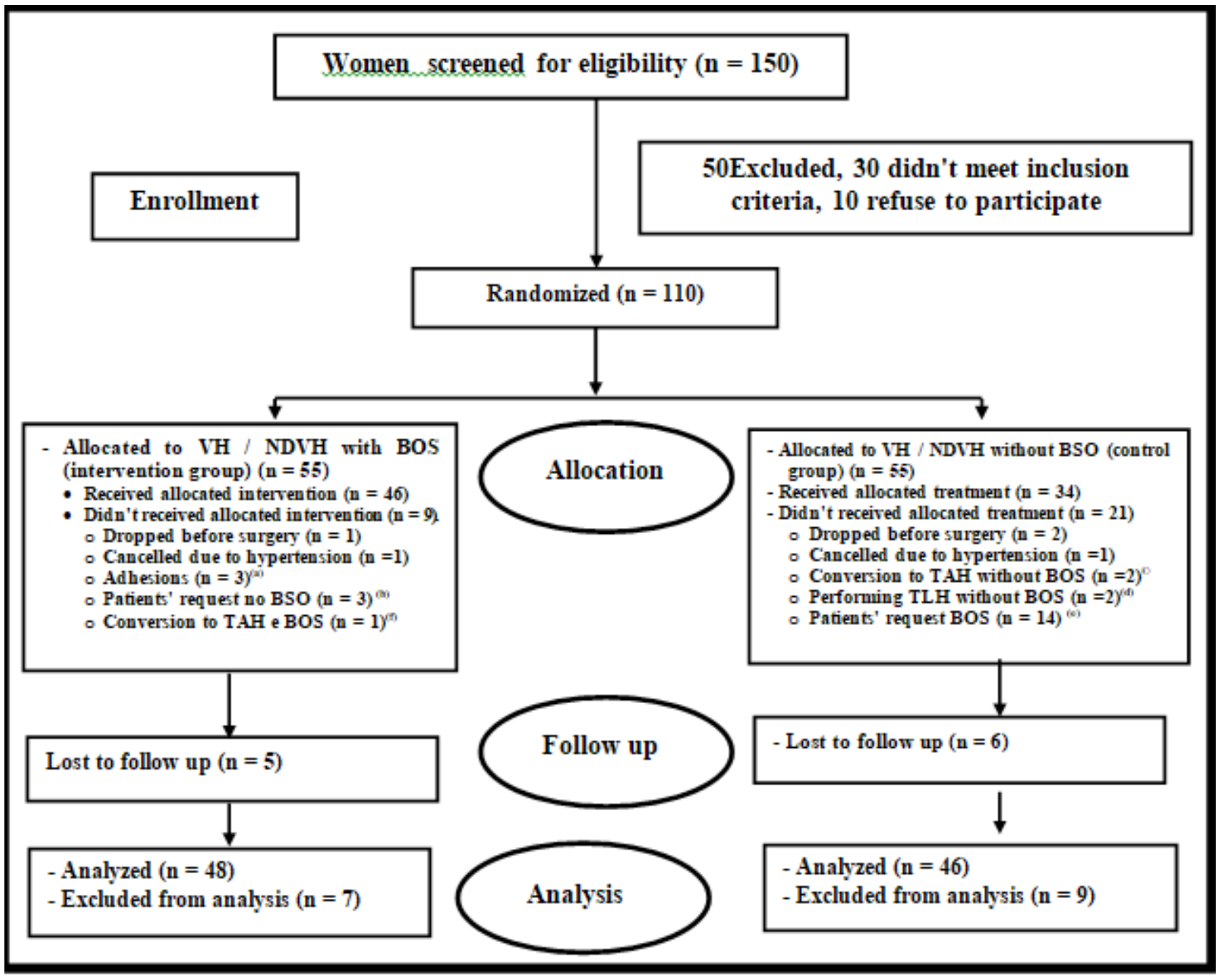

Fig. 2: CONSORT flow diagram of women included in vaginal hysterectomy with or without bilateral opportunistic salpingectomy ovarian reserve $(\mathrm{VH} \pm \mathrm{BOS} \mathrm{OR})$ trial.

Abbreviation: CONSORT: Consolidated standards of reporting trials, VH: Vaginal hysterectomy, NDVH: Non-descent vaginal hysterectomy, BOS: Bilateral opportunistic salpingectomy, TAH: Total abdominal hysterectomy, TLH: Total laparoscopic hysterectomy.

a) 3 cases in VH/NDVH with BOS group in whom BOS could not be performed due to pelvic adhesion despite completed $\mathrm{VH}$ included in this interventional group in intention to treat analysis (ITT) but included in the control group (VH/NDVH without BOS) in per protocol (PP) analysis. b) 3 cases in interventional required not to perform BOS who involved in the interventional group in ITT analysis and the control group in PP analysis. c) 2 cases in control group converted to TAH in whom no BOS performed and included in VH/NDVH control BOS group in both ITT and PP analyses. d) 2 women after randomization to control arm of this trial, senior consultant performed TLH without BOS to them and were included in the control group both in ITT and PP analyses. e) 14 cases randomized to control group but women requested to do BOS, who included in the control group as ITT analysis and the interventional group as PP analysis. f) 1 case converted to TAH in whom BSO performed and included in the interventional group in both ITT and PP analyses. 
The study revealed that table 1 presents participants baseline demographic, clinical, hormonal and three dimensional ultrasonic (3DUS) criteria and shows there are no statistically significant differences between intervention and control groups regarding age at surgery, body mass index, parity, physical status, indications for hysterectomies. Baseline AMH, FSH levels, 3DUS criteria including AFC, VI, FI, and VFI as well as calculated OvAge in years were similar in participants cohort allocated to either trial group.

Table 1: Baseline demographic, clinical, hormonal and three-dimensional ultrasound criteria of women allocated to intervention group (VH/NDVH +BOS) or control group (VH / NDVH - BOS) in VH \pm BOS OR trial.

\begin{tabular}{|c|c|c|c|c|}
\hline Variable & $\begin{array}{l}\text { Intervention group } \\
\quad(\mathrm{n}=55)\end{array}$ & $\begin{array}{l}\text { Control group } \\
(\mathrm{n}=55)\end{array}$ & $\Delta(95 \%$ C.I $)$ & P value \\
\hline Age *(year) & $45.63 \pm 8.51(35.61-49.63)$ & $44.92 \pm 9.31(36.25-48.92)$ & $0.71(-4.08,2.66)$ & $=0.67$ \\
\hline $\mathrm{BMI} *(\mathrm{~kg} / \mathrm{m} 2)$ & $29.31 \pm 9.62(22.15-38.61)$ & $28.91 \pm 9.33(21.22-39.31)$ & $0.4(-3.98,3.18)$ & $=0.82$ \\
\hline Parity* & $3.41 \pm 2.11(0-6)$ & $4.21 \pm 3.63(0-8)$ & $-0.9(-0.32,1.92)$ & $=0.16$ \\
\hline $\begin{array}{l}\text { Prior pelvi abdominal } \\
\text { surgery** } \\
\text { - None } \\
\text { - CS } \\
\text { - Others }\end{array}$ & $\begin{array}{c}3.8(69.1 \%) \\
12(21.8 \%) \\
5(9.1 \%) \\
\end{array}$ & $\begin{array}{c}35(63.6 \%) \\
14(25.5 \%) \\
6(10.9 \%) \\
\end{array}$ & $\begin{array}{l}5.5 \%(-11.90,22.45) \\
3.7 \%(-12.13,19.31) \\
1.8 \%(-10.19,13.86) \\
\end{array}$ & $\begin{array}{l}=0.54 \\
=0.64 \\
=0.75\end{array}$ \\
\hline $\begin{array}{l}\text { ASA** } \\
- \text { I } \\
\text { - II } \\
\end{array}$ & $\begin{array}{l}41(74.5 \%) \\
14(25.5 \%) \\
\end{array}$ & $\begin{array}{l}38(69.1 \%) \\
17(30.9 \%) \\
\end{array}$ & $\begin{array}{l}5.4 \%(-11.27,21.68) \\
5.4 \%(-11.27,21.68)\end{array}$ & $\begin{array}{l}=0.53 \\
=0.53\end{array}$ \\
\hline $\begin{array}{l}\text { Indications for } \\
\text { hysterectomy** } \\
\text { - POP } \geq \text { stage II POPQ } \\
\text { - DUB } \\
\text { - Fibroids } \\
\text { - CIN } \\
\text { - Others }\end{array}$ & $\begin{array}{c}18(32.7 \%) \\
22(40.0 \%) \\
9(16.5 \%) \\
3(5.4 \%) \\
3(5.4 \%) \\
\end{array}$ & $\begin{array}{c}22(40.0 \%) \\
15(27.3 \%) \\
12(21.9 \%) \\
2(3.6 \%) \\
4(7.2 \%)\end{array}$ & $\begin{array}{c}7.3 \%(-10.43,24.41) \\
12.7 \%(-4.88,29.24) \\
5.4 \%(-9.47,20.05) \\
1.8 \%(-7.57,11.53) \\
1.8 \%(-8.55,12.38)\end{array}$ & $\begin{array}{l}=0.42 \\
=0.16 \\
=0.47 \\
=0.65 \\
=0.69\end{array}$ \\
\hline $\begin{array}{l}\text { - } \mathrm{AMH}^{*}(\mathrm{ng} / \mathrm{ml}) \\
-\mathrm{FSH}^{*}(\mathrm{mIu} / \mathrm{mL}) \\
-\mathrm{AFC}^{*}(\mathrm{n}) \\
-\mathrm{VI}^{*}(\%) \\
\text { - FI } *(0-100) \\
\text { - VFI*(0-100) } \\
\text { - OVAge* (y) }\end{array}$ & $\begin{array}{c}0.69 \pm 0.51(0.11-2.31) \\
13.51 \pm 6.32(6.92-18.56) \\
4.23 \pm 1.62(2-8) \\
0.95 \pm 0.89(0.69-1.02) \\
33.38 \pm 9.31(25.81-40.18) \\
0.68 \pm 0.58(0.32-0.79) \\
43.68 \pm 8.46(34.81-48.31)\end{array}$ & $\begin{array}{c}0.78 \pm 0.61(0.12-2.82) \\
14.53 \pm 8.23(4.81-19.52) \\
4.82 \pm 1.92(2-9) \\
0.98 \pm 0.92(6.78-1.31) \\
32.61 \pm 10.23(28.23-44.31) \\
0.78 \pm 0.56(0.31-0.88) \\
44.67 \pm 9.46(35.61-49.21)\end{array}$ & $\begin{array}{c}-0.09(-0.12,0.30) \\
-1.02(-1.75,3.79) \\
-0.59(-0.81,1.26) \\
-0.03(-0.31,0.37) \\
0.77(-4.46,2.92) \\
-0.10(-0.11,0.31) \\
-0.99(-2.40,4.38) \\
\end{array}$ & $\begin{array}{l}=0.40 \\
=0.46 \\
=0.08 \\
=0.86 \\
=0.68 \\
=0.35 \\
=0.56\end{array}$ \\
\hline $\begin{array}{l}\text { Co Morbidities ** } \\
\text { - None } \\
\text { - HTN } \\
\text { - DM } \\
\text { - Others }\end{array}$ & $\begin{array}{c}36(65.4 \%) \\
8(14.5 \%) \\
6(10.9 \%) \\
5(9.1 \%)\end{array}$ & $\begin{array}{c}38(69.1 \%) \\
7(12.8 \%) \\
4(7.2 \%) \\
6(10.9 \%)\end{array}$ & $\begin{array}{l}3.7 \%(-13.54,20.64) \\
1.7 \%(-11.58,14.97) \\
1.9 \%(-10.77,14.60) \\
1.8 \%(-10.19,13.86)\end{array}$ & $\begin{array}{l}=0.68 \\
=0.79 \\
=0.75 \\
=0.75\end{array}$ \\
\hline
\end{tabular}

Abbreviations: VH: Vaginal hysterectomy, NDVH: None descent vaginal hysterectomy, BOS: Bilateral opportunistic salpingectomy, BMI: Body mass index, CS: Cesarean section, ASA: American society of anesthesiologists physical status, POP: Pelvic organ prolapse, POPQ: POP Quantification, DUB: Dysfunctional uterine bleeding, CIN: Cervical intraepithelial neoplasia, AMH: Anti-müllerian hormone, FSH: Follicular stimulating hormone, AFC: Antral follicular count, VI: Vascularization index, FI: Flow index, vFI: Vascularization flow index, DM: Diabetes mellitus, HTN: Hypertension, $\Delta$ (95\% CI): Point estimate difference with 95\% confidence interval. 
The study revealed that table 2 presented the primary as well as secondary outcomes differences between intervention and control groups. There are no statistically significant differences between participants to whom BOS is added to VH / NDVH (intervention group) from women whom did not receive BOS at VH/NDVH (control group) regarding changes $(\Delta)$ in $\mathrm{AMH}, \mathrm{FSH}, \mathrm{AFC}, \mathrm{VI}, \mathrm{FI}, \mathrm{VFI}$, OVAge at 6 to 8 months postoperatively from preoperative levels $(P>0.05)$.

Also, there is no statistically significant difference between both groups regarding $\Delta \mathrm{HB}(P=0.11)$, operative blood loss $(P=0.21)$, surgical procedures routes $[52 / 55 \quad(94.5 \%)$ in intervention group versus $48 / 55(87.2 \%)$ in control group, $p=0.18]$, operative time $(P=0.14)$, hospital stay $(p=0.41)$, postoperative resumption of normal activity $(P=0.59)$. Intended surgical approach was failed in $4(7.2 \%)$ cases in the control group where two cases performed laparoscopically and two cases were converted to TAH due to large size uteri.

In intervention group $8 / 55(14.5 \%)$ parturients didn't received allocated intervention, (3) women required to conserve their tubes, 3 cases of surgical failure to remove tubes due to adhesions, one canceled due to hypertension and one case dropped while 21 women (38\%) in the control group didn't receive the allocated treatment as 14 cases opted to do BOS, 2 cases dropped before surgery, one case canceled due to hepatic event, two cases converted to TAH but tubes were conserved and two cases undergoing TLH and tubes also conserved, so $14 / 55(25.4 \%)$ participants in controls group had BOS. The complication rate was $(23.6 \%)$ in intervention group versus $(14.5 \%)$ in control group $(P=0.22)$, most of this complication was minor event as persistent vomiting (10 cases), urinary tract infection ( 6 cases), chest infection ( 2 cases), minor grades deep venous thrombosis ( 3 cases ).

Table 2: Primary and secondary outcomes differences between intervention group (VH/NDVH + BOS) and control (VH / NDVH - BOS) in $\mathrm{VH} \pm \mathrm{BOS}$ OR trial.

\begin{tabular}{|c|c|c|c|c|}
\hline Variable & $\begin{array}{l}\text { Intervention group } \\
\quad(\mathrm{n}=55)\end{array}$ & $\begin{array}{l}\text { Control group } \\
(\mathrm{n}=55)\end{array}$ & $\Delta(95 \%$ C.I $)$ & $P$ value \\
\hline - $\Delta \mathrm{AMH}^{*}(\mathrm{ng} / \mathrm{mL})$ & $-0.12 \pm 0.21(0.03-1.92)$ & $-0.18 \pm 0.25(0.06-1.88)$ & $0.06(-0.14,0.02)$ & $=0.17$ \\
\hline$-\Delta \mathrm{FSH}^{*}(\mathrm{mIu} / \mathrm{mL})$ & $2.51 \pm 1.82(1.31-5.61)$ & $+3.12 \pm 2.23(2.11-6.52)$ & $-0.61(-0.15,1.37)$ & $=0.11$ \\
\hline$-\triangle \mathrm{AFC}^{*}(\mathrm{n})$ & $-1.21 \pm 1.62(0-4)$ & $-1.81 \pm 1.92(0-5)$ & $0.60(-0.07,1.27)$ & $=0.07$ \\
\hline$-\Delta \mathrm{VI}^{*}(\%)$ & $-0.12 \pm 0.41(0.06-0.26)$ & $-0.14 \pm 0.51(0.08-0.28)$ & $0.02(-0.15,0.19)$ & $=0.82$ \\
\hline$-\Delta \mathrm{FI} *(0-100)$ & $-0.71 \pm 2.32(0.22-6.17)$ & $-0.68 \pm 2.61(0.23-7.21)$ & $-0.03(-0.96,0.90)$ & $=0.94$ \\
\hline$-\Delta \mathrm{VFI}^{*}(0-100)$ & $-0.12 \pm 0.26(0.08-0.22)$ & $-0.16 \pm 6.22(0.06-0.19)$ & $0.04(-1.62,1.70)$ & $=0.96$ \\
\hline$-\Delta$ OVAge $*(y)$ & $+1.21 \pm 1.62(0.88-3.21)$ & $+1.12 \pm 1.83(0.86-2.91)$ & $0.09(-0.74,0.56)$ & $=0.78$ \\
\hline$-\Delta \mathrm{HB} *(\mathrm{gm} / \mathrm{dl})$ & $-1.68 \pm 1.92(0.91-2.11)$ & $-1.12 \pm 1.82(0.54-1.69)$ & $-0.56(-1.26,0.41)$ & $=0.11$ \\
\hline - Blood loss* (ml) & $350 \pm 230(220-256)$ & $310 \pm 190(150-550)$ & $50(-129.73,29.73)$ & $=0.21$ \\
\hline $\begin{array}{l}\text { - Surgical procedure** } \\
\text { - VH } \\
\text { - NDVH } \\
\text { - TAH } \\
\text { - TLH }\end{array}$ & $\begin{array}{c}17(30.9 \%) \\
35(63.6 \%) \\
1(1.8 \%) \\
\text { n.a }\end{array}$ & $\begin{array}{c}20(36.4 \%) \\
28(50.9 \%) \\
2(3.6 \%) \\
2(3.6 \%) \\
\end{array}$ & $\begin{array}{c}5.5 \%(-10.43,24.41) \\
12.7 \%(-5.53,29.79) \\
1.8 \%(-6.40,10.59) \\
\text { n.a }\end{array}$ & $\begin{array}{l}=0.42 \\
=0.17 \\
=0.54 \\
=\mathrm{n} . \mathrm{a}\end{array}$ \\
\hline $\begin{array}{l}\text {-Failure of intented } \\
\text { surgical approach** }\end{array}$ & $1^{\text {(a) }}(1.8 \%)$ & $4^{(\mathrm{b})}(7.2 \%)$ & $5.4 \%(-3.52,15.47)$ & $=0.17$ \\
\hline $\begin{array}{l}\text { - Failure of intended } \\
\text { intervention } * *\end{array}$ & $8^{(a)}(14.5 \%)$ & n.a & n.a & n.a \\
\hline
\end{tabular}




\begin{tabular}{|l|c|c|c|c|}
\hline - Operative time $(\mathrm{min}) *$ & $140 \pm 80(110-180)$ & $120 \pm 60(100-170)$ & $20(-46.72,0.72)$ & $=0.14$ \\
\hline - Complications **(c) & $13(23.6 \%)$ & $8(14.5 \%)$ & $9.1 \%(-5.75,23.59)$ & $=0.22$ \\
\hline - Hospital stay **(hours) & $20 \pm 12(6-48)$ & $18 \pm 14(6-36)$ & $-2(-6.92,2.92)$ & $=0.42$ \\
\hline $\begin{array}{l}\text {-Postoperative resumption } \\
\text { of normal activity* (days) }\end{array}$ & $8.51 \pm 6.42(4-16)$ & $9.21 \pm 7.32(4-18)$ & $-0.7(-1.90,3.30)$ & $=0.59$ \\
\hline
\end{tabular}

Abbreviations: VH: Vaginal hysterectomy, NDVH: None descent vaginal hysterectomy, BOS: Bilateral opportunistic salpingectomy, BMI: Body mass index, CS: Cesarean section, ASA: American society of anesthesiologists physical status, POP: Pelvic organ prolapse, POPQ: POP Quantification, DUB: Dysfunctional uterine bleeding, CIN: Cervical intraepithelial neoplasia, AMH:Anti-Müllerian hormone, FSH: Follicular stimulating hormone, AFC: Antral follicular count, VI: Vascularization index, FI: Flow index, vFI: Vascularization flow index, DM: Diabetes mellitus, HTN: Hypertension, $\Delta$ (95\% CI): Point estimate difference with 95\% confidence interval. n.a : not applicable.

a): one conversion to TAH with BOS. b): two conversion to TAH without BOS and two women underwent TLH without BOS c): 8 women in whom BOS did not performed allocated to the intervention group.

The presented results in tables 1 and 2 were based on an intention-to-treat analysis (ITT), however there is considerable deviation from study protocol in 30 (9 in intervention group and 21 in control group) $/ 110(27.2 \%)$ of participants resulting in 61 women underwent hysterectomy with BOS of them $60 \mathrm{VH} / \mathrm{NDVH}$ with BOS and one TAH with BOS, where 47of them were allocated to intervention group while 14 were assigned to control group. The controls were 49 cases, including 41 women were allocated to VH / NDVH without BOS, of them 34 cases received allocated treatment, 4 underwent other routes of hysterectomy without BOS while the others 3 cases didn't do surgical procedures, and the remaining 7 cases were assigned to intervention group but BOS couldn't perform in 3 patients secondary to adhesion and 3 cases opted to conserve their tubes and 1 dropped and one canceled. Owning to this deviation we performed per protocol (PP) analysis, where 61 women included in the intervention group and 49 cases involved in the control group and we found there is no statistically significant differences between both groups regards baseline criteria as well as both principal and subsidiary outcomes.

\section{DISCUSSION}

The $21^{\text {th }}$ century insights theorized that Fallopian tubes are the detrimental player in ovarian tumorigenesis especially HGSOCs. The $21^{\text {th }}$ century advances in ovarian cancer screening and management's modalities resulted only in slight improvement in OvCa morbidity and mortality. The all-cause morbidity and mortality, as well as costs of risk-reducing salpingo-oophorectomy (RRSO), are higher than consequences of ovarian conservation. All these items lead to accepting the theoretical benefits of BOS (PBS) at time of hysterectomy as OvCa riskreducing procedure (RRS) at time of pelvic surgery for benign indications and at the same time avoiding the dangerous consequences of premature surgical menopause secondary to ovarian conservation.

This prospective, multisite, randomized, concealed allocation, open-label (The only blinded persons were the sonographer and clinical pathologist), superiority trial illustrated that the adding BOS to VH / NDVH didn't impact the ovarian function (OF) and ovarian reverse (OR) more than $\mathrm{VH} / \mathrm{NDVH}$ alone at six to eight months postoperatively. Also, the adding BOS to $\mathrm{VH} / \mathrm{NDVH}$ did not add more consequences. So as surgical outcomes of both $\mathrm{VH} / \mathrm{NDVH}$ alone and $\mathrm{VH} / \mathrm{NDVH}$ with BOS were similar, the performing BOS at VH/NDVH may be beneficial at no extra cost, and only the time may prove this theoretical benefits of RRS.

As to date, no studies are evaluating the impact of adding BOS to VH/NDVH on OF and OR. We will compare our results with studies assessing effects of BOS added to TLH and TAH. Our results are in agreement with the reported finding of Morelli et al., retrospective assessment of BOS at TLH including 79 women in each arm. Also, in line with results of the prospective pilot study of Findley et al. ${ }^{[30]}$, included in total 30 women received only TLH. Moreover, in agreement with Sezik et al. ${ }^{[29]} \mathrm{RCT}$ comparing 12 total and 12 partial salpingectomy added to TAH. Also, similar results reported in equivalence powered trial of Song et al. ${ }^{[32]}$ to detect $11 \%$ decline rate in $\mathrm{AMH}$ with adding BOS including 34 women in each arm at an equivalence limits of $20 \%$ decline rate in AMH. Also, our results are in agreement with the recent RCT of Von Lieshout et al. ${ }^{[35]}$ comparing TAH / TLH with or without BOS came from Netherlands including in total 104 women and also, in 
line with Ye et al. ${ }^{[38]}$ retrospective study. The reported periods of follow up were different between these studies, were as shorter as six weeks and as longer as to one year. We elected to follow up our patients at 6 to 8 months as at this period, ovarian recovery from operative trauma is completed ${ }^{[39]}$. Song et al. ${ }^{[32]}$ reported AMH decline rate of $13 \%$ in TLH with BOS versus $11 \%$ in TLH alone $(P=0.9)$. Song et al. ${ }^{[32]}$ evaluated $\mathrm{AMH}$ alone at 3 months postoperatively while in our study the decline rate $(\triangle \mathrm{AMH} /$ preoperative level) were more, at $17 \%$ in intervention group and $23 \%$ in control group $(P=0.1)$ and also over the decline rate reported by Von Lieshout et al. ${ }^{[35]}$ which were at 6 months in intervention group 4\% and control group $(0 \%)(P=0.4)$. The variation in decline rate of AMH between trials may be due to variability in age groups of participants between trials, as well as detection kits sensitivity and the surgical techniques as well as the used technologies. All trials recording decline in OF and OR with hysterectomy whatever this significant or not and this decline is not significant when BOS was added, but the trials may differ in reported decline rate of AMH secondary to lack of AMH standardization assays. Our results were in line with studies evaluating FSH where they reported insignificant increase by adding BSO to hysterectomy ${ }^{[29,30,31,33,34]}$ as well as studies reported decline $\mathrm{AFC}^{[30,33]}$ as well as studies evaluating 3DUS vascularization indices as they reported decline in long-term to follow up ${ }^{[33]}$ as well as in short-term follow-up ${ }^{[30]}$.

Strengths in this trial includes, prospective nature, randomization, concealed allocation, sufficient power to detect a prespecified clinically significant change in $\mathrm{AMH}$ as well as assessing OF and OR through multiple item including AMH as a most reliable OR indicator used in trials ${ }^{[29-36]}$ as well as other OR indicators including FSH, AFC and recently introduced 3DUS vascularization indices as well as OVAge ${ }^{[33,34]}$. Also, we viewed the evaluation of BOS through the vaginal route significantly valuable as this covered a gap in the literature.

Limitations include lack of blinding after randomization, however this is the nature of $\mathrm{RCT}$ on surgical procedures as well as shorter period of follow up, as six months may not be sufficient time to evaluate impacts of BOS on OR, as some authors reporting variable period of recovery after ovarian surgeries starting from 6 weeks to 12 months, as reviewed by Song et al. ${ }^{[32]}$. Also, protocol violation could be a significant limiting item in this trial, as $27.2 \%$ of women did not receive allocated treatment ,however, this is nature of RCT on surgical procedures as well as on such debatable topics such our topic. Moreover, we performed ITT as well as PP analyses, and both were in agreement regarding primary and secondary outcomes measures of the trial. Also, 10\% lost to follow up could be viewed as a limitation, however, failed to follow up according to ITT and PP analyses were equally divided between the intervention as well as the control group and so selective loss to follow up is unlikely. Additional limitation includes evaluation of surrogate parameters of OF and OR rather than considering menopausal symptoms onset, however to conducting such trial, this is a challenging task as it needs very long time as well as larger sample size ${ }^{[21,40]}$. Also, we may be viewing the skill of adding BOS to VH/NDVH couldn't be generalized as well as variability in performing BOS with sutures, monopolar and bipolar energy could be the cause of higher decline rate of AMH compared to Song et al. ${ }^{[32]}$ as well as Von Lieshout et al. ${ }^{[35]}$. Whom utilized vessel selling instruments, however, what we did is, what could be applied to low resource countries, like us in Egypt. The population cohorts of this trial could be evaluated later on regards ovarian reserve, menopausal symptoms onset, adnexal surgical consequences as well as ovarian cancer risk reduction. Studies evaluating detrimental effects of different techniques and technologies on ovarian reserve and surgical procedures profiles the vaginal route might be needed.

\section{CONCLUSION}

Bilateral opportunistic salpingectomy (BOS)at time of vaginal hysterectomy $(\mathrm{VH}) /$ non-descent vaginal hysterectomy (NDVH)did not have detrimental impact on ovarian function and ovarian reserve as evaluated by change in AMH, FSH, AFC, VI, FI, VFI, OVAge as well as didn't increase surgical risks over that happened with VH / NDVH alone. This suggested that BOS at time of $\mathrm{VH} / \mathrm{NDVH}$ for benign indications as a mean of ovarian cancer risk reduction in average-risk women is a safe option despite that thus benefit remains to be elucidated.

\section{CONFLICT OF INTEREST}

There are no conflicts of interests.

\section{REFERENCES}

1. Ferlay J, Soerjomataram I, Dikshit R, Eser S, Mathers C, Rebelos M, et al. Cancer incidence and mortality worldwide: sources, methods and major patterns in GLOBOCAN 2012. Int $\mathrm{J}$ Cancer 2015; 136: E359-86.

2. Torre LA, Bray F, Siegel RL, Ferlay J, LortetTieulent J, Jemal A. Global cancer statistics, 2012. CA Cancer J Clin 2015; 65: 87-108. 
3. National Cancer Institute. Surveillance, Epidemiology, and End Results Program, SEER Stat Fact Sheets: Ovary Cancer. Available at: http://seer.cancer.gov/statfacts/html/ovary.html. Accessed May 20, 2018.

4. Piek JM, van Diest PJ, Zweemer RP, et al. Dysplastic changes in prophylactically removed fallopian tubes of women predisposed to developing ovarian cancer. J Pathol. 2001; 195: 451-456.

5. Piek JM, Verheijen RH, Kenemans $P$, Massuger LF, Bulten H, van Deist PJ. BRCA12-/related ovarian cancers are of tubal origin: a hypothesis. Gynecol Oncol. 2003; 90:491.

6. Kuman RJ, Shih IM. Molecular pathogenesis and extraovarian origin of epithelial ovarian cancer-shifting the paradigm. Hum Pathol. $2011 ; 42$ : 918-931.

7. Medeiros F, Muto MG, Lee Y, Elvin JA, Callahan MJ, Feltmate C, et al. The tubal fimbria is a preferred site for early adenocarcinoma in women with familial ovarian cancer syndrome. Am J Surg Pathol. 2006; 30:230-6.

8. Rabban JT, Garg K, Crawford B, Chen LM, Zaloudek CJ. Early detection of high-grade tubal serous carcinoma in women at low risk for hereditary breast and ovarian cancer syndrome by systematic examination of fallopian tubes incidentally removed during benign surgery. Am J Surg Pathol. 2014; 38 : 729 - 42.

9. Reade CJ, McVey RM, Tone AA, et al. The fallopian tube as the origin of high-grade serous ovarian cancer: review of a paradigm shift. J Obstet Gynaecol Can. 2014; $36: 133140$.

10. GOC statement regarding salpingectomy and ovarian cancer prevention. Available at: https://www.g-o-c.org/uploads/11 sept 15 gocevidentiarystatement_final_en.pdf. Accessed May 18, 2018.

11. SGO Clinical Practical Statement: Salpingectomy for ovarian cancer prevention. Available at : https://www.sgo.org/clinical-practice/guidelines/ sgo-clinical-practice-statement-salpingectomyfor-ovarian-cancer-prevention/. Accessed May $18,2018$.
12. Committee on Gynecologic Practice. Salpingectomy for ovarian cancer prevention. Committee opinion no. 620. Obstet Gynecol $2015 ; 125: 279-81$.

13. McAlpine JN, Hanley GE, Woo MM, Tone AA, Rozenberg N, Swenerton KD, et al. Ovarian cancer research program of British Columbia. Opportunistic salpingectomy: uptake, risks, and complications of a regional initiative for ovarian cancer prevention. Am J Obstet Gynecol. 2014;210:471.e111-

14. Kapurubandara S, Qin V, Gurram D, Anpalagan A, Merkur H, Hogg $\mathrm{R}$, et al. Opportunistic bilateral salpingectomy during gynecological surgery for benign disease: a survey of current Australian practice. Aust NZ J Obstet Gynecol. 2015; 55 : 606-11.

15. Chene $\mathrm{G}$, de Rochambeau B, Le Bail-Carval K, Beaufils E, Chabert P, Mellier G, et al. Current surgical practice of prophylactic and opportunistic salpingectomy in France. Gynecol Obstet Fertil. 2016; 44 : 377- 84.

16. Yoon SH, Kim SN, Shim SH, Kang SB, Lee SJ. Bilateral salpingectomy can reduce the risk of ovarian cancer in the general population: a metaanalysis. Eur J Cancer. 2016; 55: 38-46.

17. Darelius A, Lycke M, Kindblom JM, Kristjansdottir B, Sundfeldt K, Strandell A. Efficacy of salpingectomy at hysterectomy to reduce the risk of epithelial ovarian cancer: a systematic review. BJOG. 2017; 124 : 880-9.

18. Falconer H, Yin L, Gronberg H, Altman D. Ovarian cancer risk after salpingectomy: a nationwide population-based study. J Natl Cancer Inst. 2015 Jan 27; 107. DOI: https://doi.org/10.1093/jnci/ dju410. Print 2015 Feb.

19. Lessard-Anderson CR, Handlogten KS, Molitor RJ, Dowdy SC, Cliby WA, Weaver AL, et al. Effect of tubal sterilization technique on risk of serous epithelial ovarian and primary peritoneal carcinoma. Gynecol Oncol. 2014 Dec; $135: 423-427$.

20. Madsen C, Baandrup L, Dehlendorff C, Kjaer SK. Tubal ligation and salpingectomy and the risk of epithelial ovarian cancer and borderline ovarian 
tumors: a nationwide case-control study. Acta Obstet Gynecol Scand. 2015 Jan; 94: 86-94.

21. Clinical trial registration. https://clinicaltrials.gov/ ct2/show/ NCT03045965? Cond = Opportunistic + salpingectomy \& rank $=1$.assessed 15 September 2018.

22. Nieboer TE, Johnson $\mathrm{N}$, Lethaby A, et al. Surgical approach to hysterectomy for benign gynecological disease. Cochrane Database Syst Rev. 2009: CD003677.

23. Robert M, Cenaiko D, Sepandj J, Iwanicki S. Success and complications of salpingectomy at the time of vaginal hysterectomy. J Minim Invasive Gynecol. 2015; 22 : 864-9.

24. Lamblin G, Meysonnier C, Moret S, Nadaud B, Mellier G, Chene G. Opportunistic salpingectomy during vaginal hysterectomy for a benign pathological condition. Int Urogynecol J (2018) 29:715-721 DOI 10.1007/s00192-017-3418-0

25. Rosanne $\mathrm{M}$ and Kho, MD. Prophylactic bilateral salpingectomy at vaginal hysterectomy: time for a policy? NOVEMBER 2017 American Journal of Obstetrics \& Gynecology 503 - 504.

26. American College of Obstetricians and Gynecologists. Choosing the route of hysterectomy for benign disease. ACOG Committee opinion no. 444. Obstet Gynecol 2009;114:1156-8

27. Antosh DD, High R, Brown HW, et al. Feasibility of prophylactic salpingectomy during vaginal hysterectomy. Am J Obstet Gynecol 2017;217:605.e1-5.

28. Cadish LA, Shepherd JP, Barber EL, Ridgeway B. Risks and benefits of opportunistic salpingectomy during vaginal hysterectomy: a decision analysis. Am J Obstet Gynecol 2017; 217:603.e1-6

29. Sezik M, Ozkaya O, Demir F, Sezik HT, Kaya H. Total salpingectomy during abdominal hysterectomy: effects on ovarian reserve and ovarian stromal blood flow, J. Obstet. Gynecol. Res. 33 (2007) 863-869, http://dx.doi.org/10.1111/j. 14470756.2007.00669-.x

30. Morelli M, Venturella R, Mocciaro R, Di Cello A, Rania E, Lico D, D’Alessandro P, Zullo F. Prophylactic salpingectomy in premenopausal low-risk women for ovarian cancer: primum nonnocere, Gynecol. Oncol. 129 (2013) 448-451, http://dx.doi.org/10.1016/j.ygyno.2013.03.023.

31. Findley AD, Siedhoff MT, Hobbs KA, Steege JF, Carey ET, McCall CA, Steiner AZ. Short-term effects of salpingectomy during laparoscopic hysterectomy on ovarian reserve: a pilot randomized controlled trial, Fertil. Steril. 100 (2013) 1704-1708, http://dx.doi.org/10.1016/j. fertnstert.2013.07.1997.

32. Song T, Kim MM-LKMK, Jung YW, Yun BS, Seong SJ, Kwon S, Impact of opportunistic salpingectomy on anti-Müllerian hormone in patients undergoing laparoscopic hysterectomy: a multicentre randomized controlled trial, BJOG Int. J. Obstet. Gynecol. (2016) 17, http://dx.doi. org/10.1111/ 14710528.14182-.

33. Venturella R, Lico D, Borelli M, Imbrogno MJ, Cevenini G, Zupi E, Zullo F, Morelli M. 3 to 5 years later: long-term effects of prophylactic bilateral salpingectomy on ovarian function, J. Minim. Invasive Gynecol. 24 (2017) 145-150, http://dx.doi.org/10.1016/j.jmig.2016.08.833.

34. Venturella R, Morelli M, Lico D, Cello AD, Rocca M, Sacchinelli A, Mocciaro R, D'Alessandro P, Maiorana A, Gizzo S and Zullo F. Wide excision of soft tissues adjacent to the ovary and fallopian tube does not impair the ovarian reserve in women undergoing prophylactic bilateral salpingectomy: results from a randomized, controlled trial. Fertil Steril. 2015 Nov;104(5):13329-. doi: 10.1016/j. fertnstert.2015.08.004. Epub 2015 Aug 31.

35. Van Lieshout LAM, Pijlmanb B, Vosc MC, de Groot MJM, Houtermane S, Corppus SFPJ, Harmsen MG, Vandenputa I, Pieka JMJ. Opportunistic salpingectomy in women undergoing hysterectomy: Results from the HYSTUB randomized controlled trial. Maturitas 107 (2018) 16-.

36. Venturella R, Lico D, Sarica A, Falbo MP, Gulletta E, Morelli M, et al. OvAge: a new methodology to quantify ovarian reserve combining clinical, biochemical and 3D-ultrasonographic parameters. J Ovarian Res. 2015; 8: 21.

37. Van Rooij IAJ, Broekmans FJM, Scheffer GJ, Looman CWN, Habbema JDF, De Jong FH, Fauser BJCM, Themmen APN, Te Velde ER. 
Serum antimüllerian hormone levels best reflect the reproductive decline with age in normal women with proven fertility: a longitudinal study, Fertil. Steril. 83 (2005): 979-987, http:// dx.doi. org/10.1016/ j.fertnstert.2004.11.029

38. Ye XP, Yang YZ, Sun XX. A retrospective analysis of the effect of salpingectomy on serum Anti Müllerian hormone level and ovarian reserve, Am. J. Obstet. Gynecol. 212 (2015) 53, http://dx.doi. org/10.1016/j.ajog.2014.07.027 (e153-. e10).
39. Atabekoglu C, Taskin S, Kahraman K, Gemici A, Taskin EA, Ozmen B, Berker B, Sonmezer M. The effect of total abdominal hysterectomy on serum anti-Mullerian hormone levels: a pilot study, Climacteric 15 (2012) 393-397, http:// dx.doi.org/ 10.3109 / 13697137.2011 .642426 .

40. Manchanda R, Menon U. Opportunistic bilateral salpingectomy (OBS) for the prevention of ovarian cancer should be offered in the context of a clinical trial. BJOG 2016;123:463. 